\title{
Orally disintegrating olanzapine review: effectiveness, patient preference, adherence, and other properties
}

This article was published in the following Dove Press journal:

Patient Preference and Adherence

31 January 2012

Number of times this article has been viewed

\author{
William Montgomery' \\ Tamas Treuer ${ }^{2}$ \\ Jamie Karagianis ${ }^{3}$ \\ Haya Ascher-Svanum ${ }^{4}$ \\ Gavan Harrison ${ }^{5}$ \\ 'Global Health Outcomes, Eli Lilly \\ and Company, Sydney, Australia; \\ ${ }^{2}$ Emerging Markets Business Unit \\ (Neuroscience), Eli Lilly and \\ Company, Budapest, Hungary; \\ ${ }^{3}$ Eli Lilly and Company, \\ Indianapolis, IN, USA; ${ }^{4} \mathrm{Global}$ \\ Health Outcomes, Eli Lilly and \\ Company, Indianapolis, IN, USA; \\ ${ }^{5}$ Asia-Pacific Medical Communications, \\ Eli Lilly and Company, Sydney, Australia
}

Correspondence: William Montgomery Eli Lilly Australia, 112 Wharf Road, West Ryde, NSW 21 I4, Australia

Tel +6I 293254335

Fax +6I 293254334

Email montgomery_bill@lilly.com

\begin{abstract}
Orally disintegrating olanzapine (ODO) is a rapid-dissolving formulation of olanzapine which disintegrates in saliva almost immediately, developed as a convenient and adherence-enhancing alternative to the standard olanzapine-coated tablet (SOT). Clinical studies, which form the basis of this review, have shown ODO and SOT to have similar efficacy and tolerability profiles. However, ODO appears to have a number of advantages over SOT in terms of adherence, patient preference, and reduction in nursing burden. Overall, the existing clinical data suggests that compared to SOT, ODO is not only well-suited for difficult-to-treat, agitated, and/or nonadherent patients but, due to its potential ability to improve adherence and greater patient preference, may also be an appropriate formulation for the majority of patients for which olanzapine is the antipsychotic of choice.
\end{abstract}

Keywords: orodispersible formulation, orally disintegrating, olanzapine, atypical antipsychotics, patient adherence, preference, schizophrenia, bipolar disorder

\section{Introduction}

Olanzapine is an efficacious and well-tolerated atypical antipsychotic indicated for the treatment of schizophrenia and acute manic or mixed episodes, along with maintenance therapy in bipolar disorder and (in some countries) related psychiatric disorders. ${ }^{1-3}$ Consistent with the findings from large comparative clinical trials and observational studies, ${ }^{4-8}$ olanzapine has been found to be comparable or superior to other atypical antipsychotic medications in meta-analyses of head-to-head studies using a variety of efficacy/effectiveness and safety/tolerability outcomes. ${ }^{1,2}$ Notwithstanding potential weight gain as an important consideration associated with olanzapine treatment, this medication has a favorable risk/benefit profile that has led to it being extensively utilized worldwide. ${ }^{9}$

In order to optimize the utility of olanzapine in patient groups with different needs and preferences, a number of formulations have been developed. In addition to short- and long-acting injectable formulations, olanzapine is commercially available in two primary oral dosage forms, standard olanzapine-coated tablets (SOT) and orally disintegrating olanzapine (ODO; otherwise known as orodispersible tablets [ODT]). Oral olanzapine is also available as granules in Japan. SOT is marketed under the trade name Zyprexa ${ }^{\circledR}$ and ODO under the name Zyprexa ${ }^{\circledR}$ Zydis $^{\circledR}$ (where "Zydis" refers to the patented freeze-dried orodispersible technology of Catalent Pharma Solutions, Somerset, NJ) or Zyprexa ${ }^{\circledR}$ Velotab $^{\mathrm{TM}}$ (in Europe). The majority of clinical studies concerning the efficacy/effectiveness, safety, and health outcomes associated with olanzapine treatment have been conducted with SOT. With few exceptions mentioned 
in this review, almost all randomized controlled trials (RCTs) of olanzapine have used SOT.

Although liquid and injectable (rapid-acting and longacting depot) antipsychotics are alternatives to standard tablets in various clinical situations, for example in the acute setting or where patients have adherence issues, these formulations have limitations. Patients may spit out liquid medication or find injectable medication unacceptable or contraindicated. Despite the clinical utility of olanzapine in standard tablet form (ie, SOT), this formulation also has a number of limitations inherent to all standard tablets in the treatment of chronic mental illness. These include administration in situations where the patient is acutely agitated, use in patients with concomitant medical conditions where there is difficulty in swallowing, taste which some patients may find disagreeable, or the need for fluids to ingest which may not be available and may draw unwanted attention to the patients' condition. Most importantly, treatment nonadherence (particularly surreptitious/deliberate avoidance) is a major problem in patients with mental illnesses such as schizophrenia. Up to $80 \%$ of patients are at least partially nonadherent to antipsychotic treatment at some point during their illness, ${ }^{10}$ which is, in turn, a leading contributor to treatment failure, relapse, and hospitalization. ${ }^{1,11,12}$ Moreover, given the patient population, which often includes persons with limited insight into their illness, any measures that may help enhance palatability or acceptance of medication is likely to aid treatment adherence and persistence and improve long-term outcomes for the patient. Thus, although rapid-acting intramuscular formulations are effective, for example in treating acutely agitated patients, oral medications can be used in this situation and have been shown to be preferred by patients. ${ }^{13}$ A number of treatment guidelines for the management of acutely agitated and aggressive patients recommend that the oral route of administration is preferred and that oral antipsychotics like SOT and ODO, with or without benzodiazepines, be considered prior to the use of intramuscular antipsychotic formulations. ${ }^{14,15}$ As oral treatments are less invasive than injections, this is consistent with the need to establish a good initial therapeutic alliance that predicts a positive attitude towards medication, which in turn predicts adherence. ${ }^{16}$

To some extent, as with liquid formulations of antipsychotics, a palatable and effective rapidly-disintegrating oral formulation which cannot be sequestered ("cheeked") for later expulsion (in other words, for which ingestion can be verified) may overcome some or all of the difficulties associated with standard tablets and injectables in individual patients.
Thus, ODO (at least in Zydis form) provides the benefits of a liquid medication in a solid dosage form and is the reason ODO was developed. Prescription data in the UK supports the proposition that ODO may be used as an alternative to depot formulations in patients with adherence issues. ${ }^{17}$ Analysis of a number of recent trials in patients with schizophrenia, where the clinician was given the option of prescribing ODO or SOT, show that ODO was preferentially used in patients who were sicker/more acutely ill (see below). It should be noted that other nonproprietary (generic) orally dissolving formulations of olanzapine, which consist of loosely-compressed rather than lyophilized wafers, are marketed in a number of countries around the world. However, unless otherwise specified, ODO referred to in this article is the proprietary Zyprexa Zydis/Velotab forms manufactured through freezedried technology and marketed by Eli Lilly and Company (Indianapolis, IN).

The purpose of this article is to provide an overview of ODO characteristics and review the available data on its clinical utility (physical properties, effectiveness, safety, adherence, nursing burden, and preference), particularly on the basis of recent studies. For a comprehensive review of earlier ODO studies, the reader is also referred to the review article by San et al. ${ }^{18}$ All studies of ODO uncovered from a PubMed search (as at November 8, 2011; English language articles only) using the terms "orally disintegrating olanzapine," "orally dissolving olanzapine," "orodispersible olanzapine," “zyprexa zydis," “ODT olanzapine," "ODO olanzapine," and "velotab" and references therein, as well as currently unpublished Eli Lillysponsored studies, were considered for inclusion in this review if they were of reasonable sample size and of sufficient rigor in design to allow meaningful interpretation.

\section{ODO characteristics}

A number of orally disintegrating tablet or wafer formulations have recently been developed with the advantage of dissolving rapidly in the oral cavity without the need for liquids ${ }^{19}$ and masking the taste of the medication. ${ }^{20}$ It is to this class of medication delivery product that ODO belongs. Produced by freeze-drying through the Zydis method, ODO consists of olanzapine as the active ingredient entrapped within a matrix of fast-dissolving carrier material. ${ }^{21}$ It is administered by placing it on the tongue, where it rapidly disintegrates.

ODO tablets are yellow, round, and debossed with the tablet strength. Each ODO tablet contains the olanzapine equivalent of $5 \mathrm{mg}(16 \mu \mathrm{mol}), 10 \mathrm{mg}(32 \mu \mathrm{mol}), 15 \mathrm{mg}(48 \mu \mathrm{mol})$, or $20 \mathrm{mg}(64 \mu \mathrm{mol})$. ODO also contains the following 
inactive ingredients: gelatin, mannitol, aspartame, sodium methylparaben, and sodium propylparaben. Phenylalanine $(0.34,0.45,0.67$, or $0.90 \mathrm{mg}$ per $5,10,15$, or $20 \mathrm{mg}$ ODO tablet, respectively) is also included (US prescribing information available from: http://pi.lilly.com/us/zyprexa-pi.pdf).

ODO tablets dissolve in human saliva in seconds. For example, in a pilot study of eleven patients, where the first measured time points were 15 seconds for initial disintegration and 60 seconds for complete disintegration, Chue et al found that the mean time to initial disintegration was 15.78 seconds and mean time to complete disintegration was 0.97 minutes. $^{22}$ As some medications in orally disintegrating formulation undergo pregastric absorption (from saliva in the mouth, pharynx, and esophagus), bioavailability of such orodispersible forms has been found to be considerably greater than their standard dosage forms. ${ }^{21}$ Although oral absorption may be enhanced with ODO, this has not been found to result in a different pharmacokinetic profile relative to SOT. While a number of studies have demonstrated more rapid onset of absorption of olanzapine with ODO and earlier measurable and higher plasma concentrations within the first 1-2 hours compared with SOT, the clinical relevance of these differences is unclear. It has been suggested that ODO may have a faster onset of action and be more appropriate for the treatment of acute agitation in schizophrenia or bipolar disorder. ${ }^{23}$ However, ODO was found to be bioequivalent to the same dosage of SOT with the maximum observed concentration and time taken to reach maximum observed concentration being almost indistinguishable between the two formulations. ${ }^{23-25}$ For example, although Bergstrom et al found more rapid initial absorption into the blood of olanzapine from ODO, the difference in means between ODO and SOT for maximum plasma concentration and area under the curve of plasma concentration over time were all $<10 \%$ and the $90 \%$ confidence intervals for the ratio of mean values were within the standard bioequivalence limits of $0.80-1.25$. Absorption rate constants for the two formulations have also not been found to be considerably different. ${ }^{23}$

\section{Comparative dissolution}

Generic mouth-disintegrating forms of olanzapine are manufactured by several different companies using different formulations and processes. In a recent in vitro study by Hobbs et al, differences in disintegration and dissolution time of the active ingredient (along with a number of other parameters) of twelve types of mouth-disintegrating olanzapine tablets (including ODO), along with Risperdal $\mathrm{M}-\mathrm{Tab}^{\circledR}$ as a comparator, were investigated using automated dissolution test equipment. ${ }^{26}$ While this study is the subject of a manuscript in preparation and will not be described here in detail, ODO disintegrated and dissolved (ie, released active ingredient) more rapidly in a solution of synthetic saliva than any of the generic tablets. Some of the generic tablets were also found to leave a coarse insoluble residue from the excipients used. Hobbs et al concluded that these different disintegration times, excipient content, and tablet residue could influence mouth feel and taste which may in turn impact adherence to treatment; however, this assumption has yet to be tested in vivo.

\section{Clinical studies}

Effectiveness of a medication takes into consideration a variety of factors such as its efficacy and safety, patient adherence, and the ease of use for patients. As summarized in Table 1, a number of studies have examined these factors in relation to ODO and their results will be discussed in detail below.

Of the fifteen clinical studies assessing the efficacy/ effectiveness of ODO, two are RCTs: one is a double-blind parallel-arm trial assessing the safety of ODO compared with SOT as its primary objective ${ }^{27}$ and the other is an openlabel crossover study assessing patient preference for ODO compared with SOT. ${ }^{28}$ Four observational cohort studies have also compared patients taking ODO and SOT. ${ }^{29-32}$ Additionally, one observational cohort study compared ODO with standard oral therapies, ${ }^{13}$ while others compared ODO with risperidone oral solution, ${ }^{33}$ as well as intramuscular olanzapine and haloperidol. ${ }^{34}$ One small retrospective open-label study compared ODO and SOT, with randomization to antipsychotic but chronological assignment to ODO or SOT. ${ }^{35}$ There are also a number of single-arm studies assessing ODO: a small pilot study by Chue et al examined the safety and acceptance of ODO in patients already stabilized on SOT, ${ }^{22}$ a 4 -week study by Hori et al assessed the efficacy, safety, and acceptance of ODO in patients with first-episode psychosis, ${ }^{36}$ a 6-week observational study assessed ODO in a French cohort of psychotic patients, ${ }^{37}$ and a 6-week study by Kinon et al examined the efficacy, safety, and acceptance of ODO in a population of nonadherent patients. ${ }^{38} \mathrm{~A}$ singlearm retrospective observational study of ODO in Belgium has also been conducted. ${ }^{39}$

\section{Efficacy/effectiveness}

The majority of studies investigating the efficacy/effectiveness of ODO have been conducted in patients with schizophrenia spectrum disorders and have used a number of 


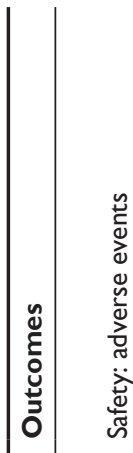
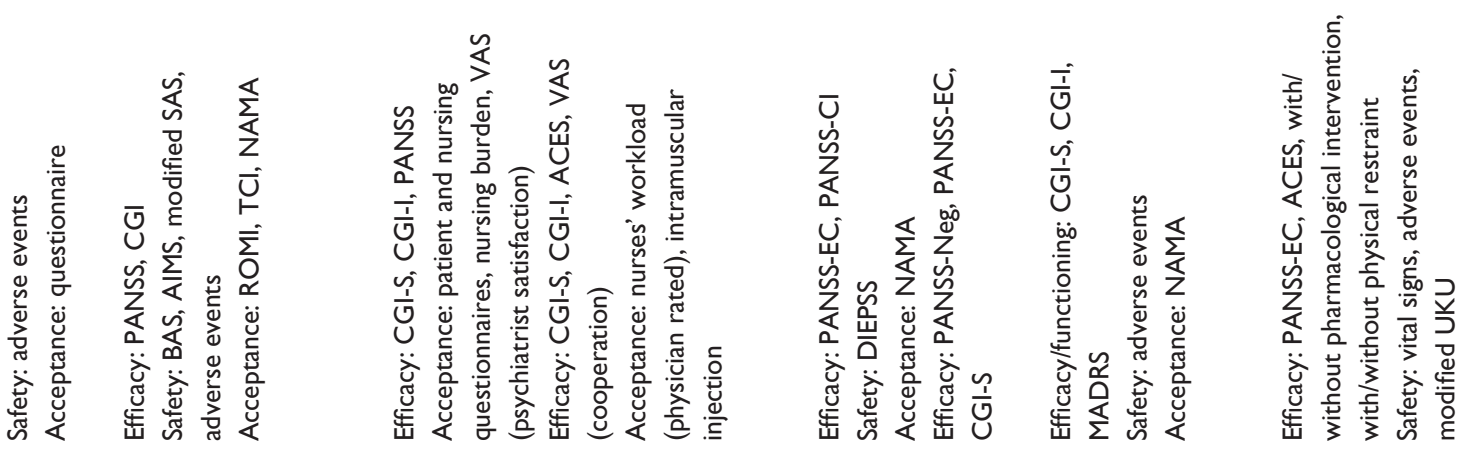

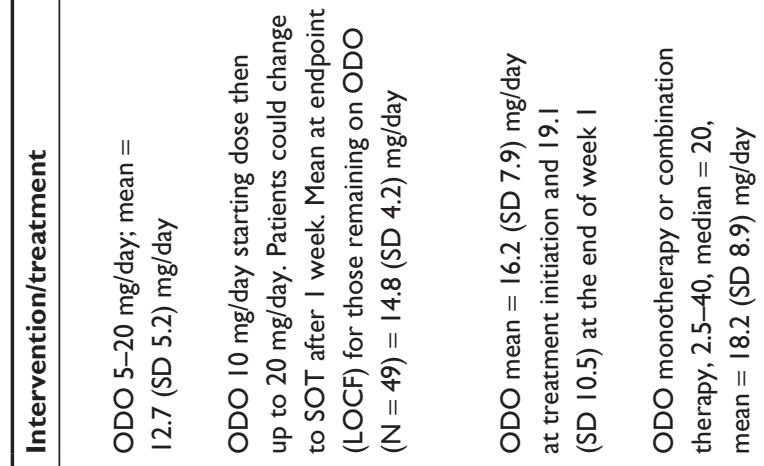
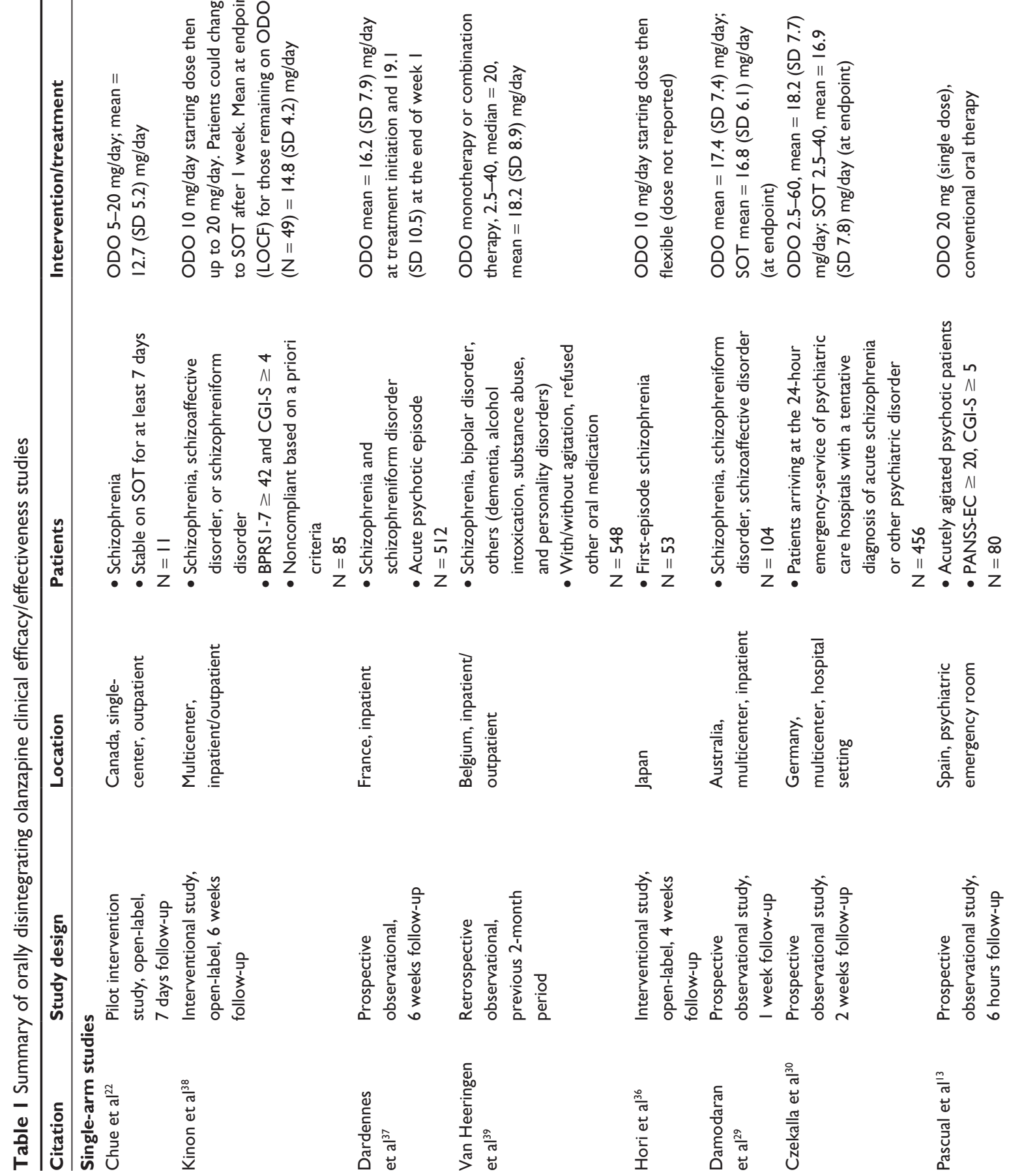

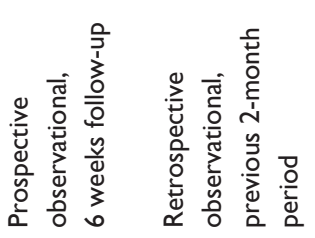

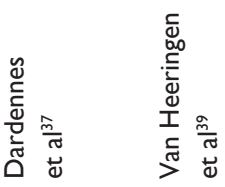

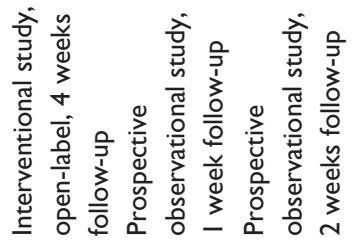

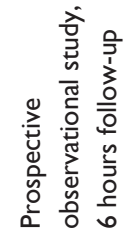

$\frac{m}{\frac{m}{\pi}}$ 

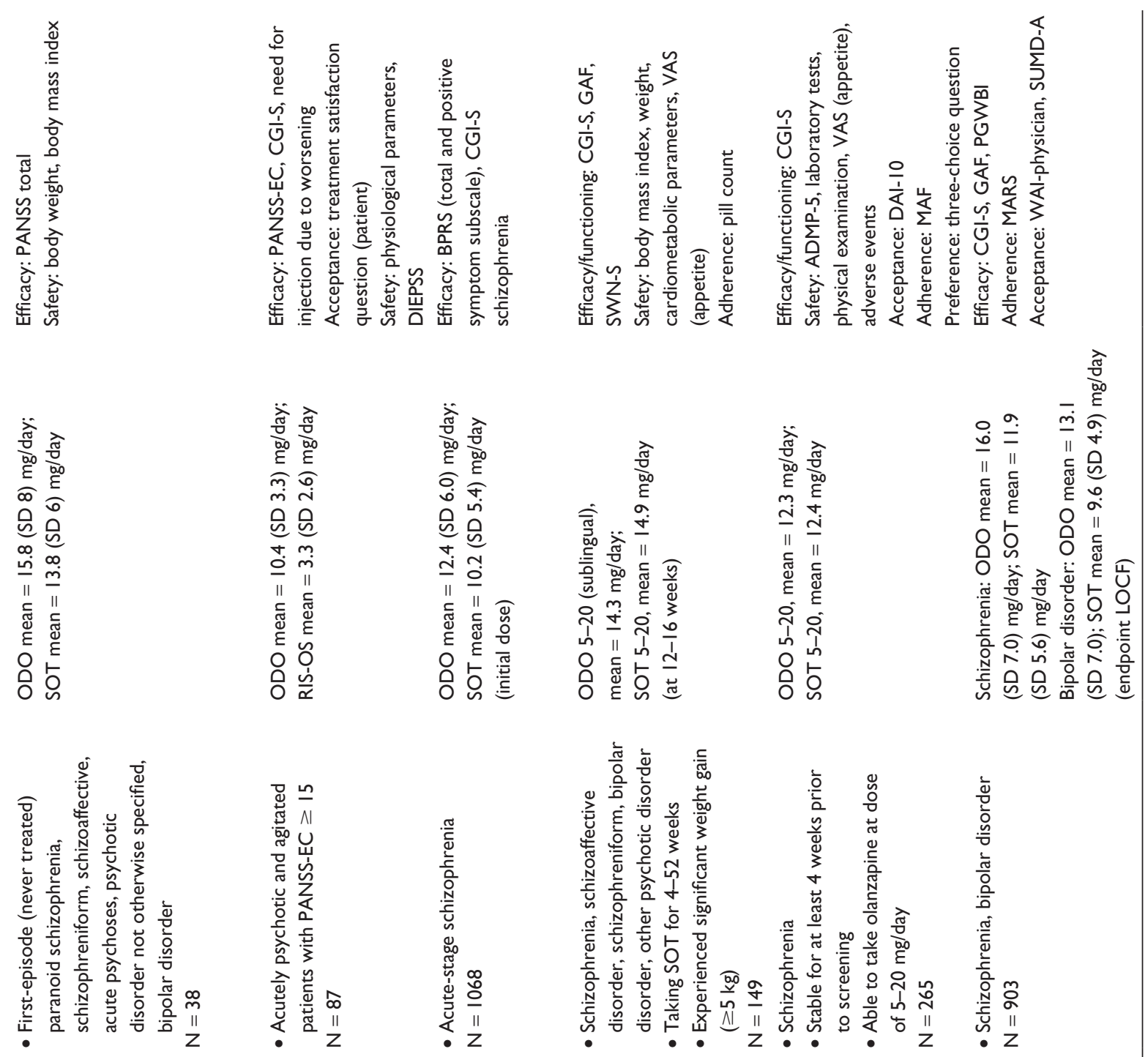

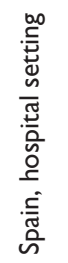
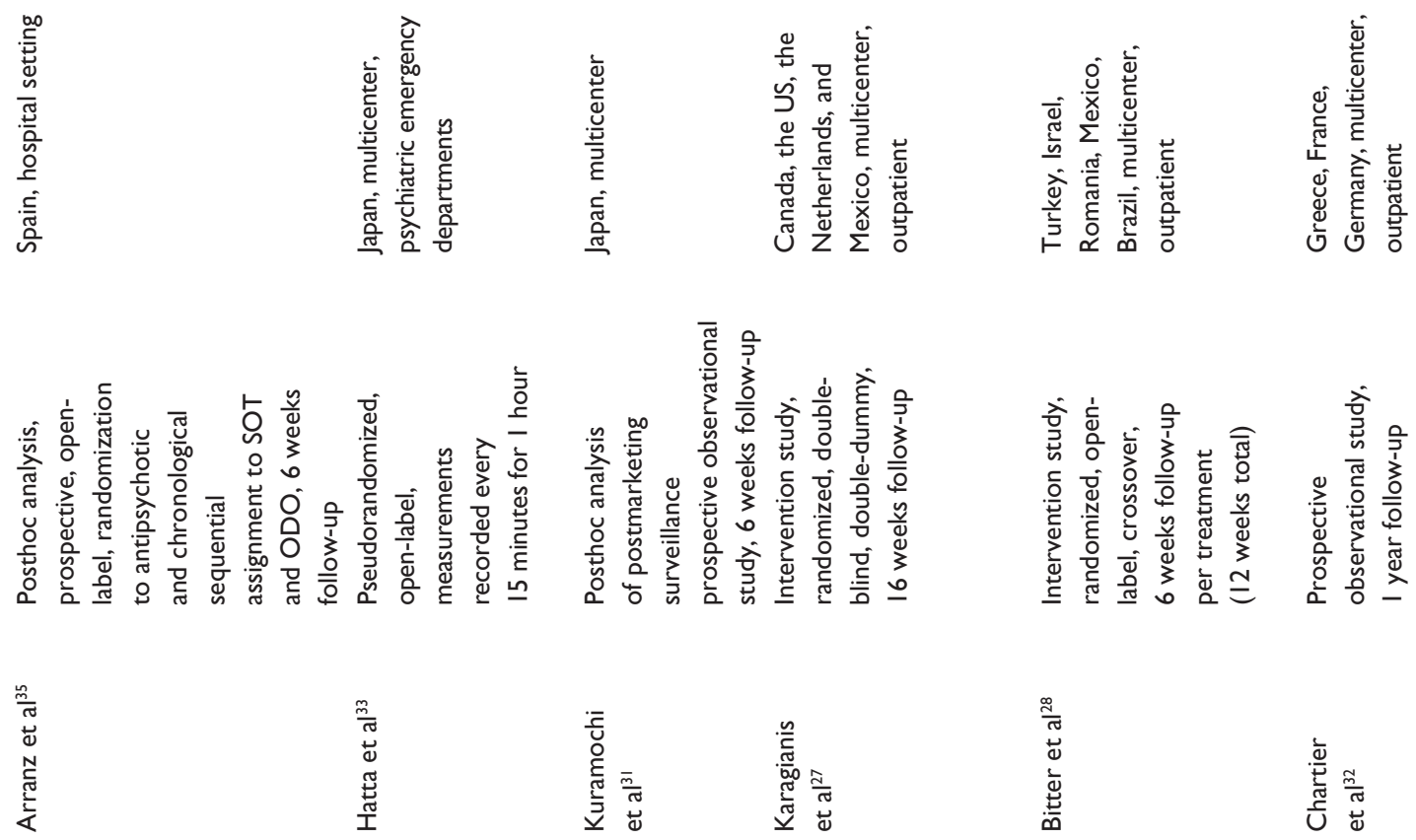

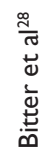

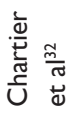




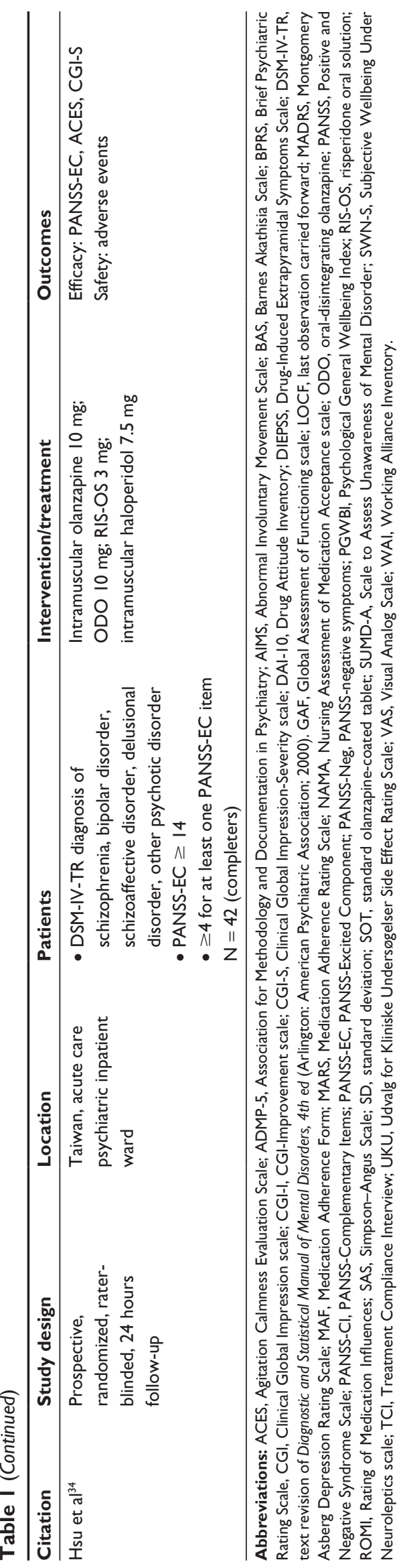

standard clinician-rated instruments as measures of symptom improvement - most commonly, the Positive and Negative Symptom Scale (PANSS) and its subscales ${ }^{40}$ and the Clinical Global Impression (CGI) scale. ${ }^{41}$ These studies have been conducted as either open-label assessments of ODO alone (single-arm studies) or comparison of ODO to SOT, or, in a few cases, to other typical and/or atypical antipsychotics (comparative studies). The studies from each of these categories are described separately below.

\section{Single-arm studies}

Results from single-arm studies show that following the initiation of therapy with ODO, a substantial improvement in the symptoms of schizophrenia is observed within a short time-frame ( $<1$ week). In the 6-week study of acutely ill, previously nonadherent patients $(\mathrm{N}=85)$ conducted by Kinon et al, ${ }^{38}$ patients were started on ODO but could switch to SOT after 1 week ( 24 patients switched to SOT, while 49 patients remained on ODO). The study showed a significant improvement in PANSS total score by week 1 , which was maintained to the end of the study (PANSS total score change to endpoint, last observation carried forward: $-24.41, P<0.001$ ), while an improvement in CGI-Improvement score occurred between day 2 and week 6 (endpoint 2.74, $P<0.001$ ).

Studying 512 acutely psychotic French patients predominantly diagnosed with schizophrenia and treated with ODO in a hospital setting, Dardennes et al found that the majority of patients treated with ODO had been previously poorly adherent $(51.0 \%)$ with a poor attitude towards medication or had refused medication (74.6\%). ${ }^{37}$ During this 6-week study, PANSS total score decreased (last observation carried forward) from 106.9 to 65.7 and the proportion of patients reporting being very satisfied with ODO increased from $22.9 \%$ to $29.5 \%$. Statistical significance for these results was not reported. ${ }^{37}$

Van Heeringen et al conducted a retrospective study in a cohort of 548 Belgian psychiatric patients diagnosed with various conditions, most of whom were not willing to take their medication and were agitated $(82.1 \%) .{ }^{39}$ The study aimed to determine if ODO had an impact on nursing workload or reduced the need for intramuscular drugs (thus reducing potential for needle stick injury, reducing the amount of patient monitoring, and helping to build the therapeutic alliance between patient and healthcare professionals). On the day following initiation, significant improvements in agitation and degree of cooperation were observed. The most common reasons cited for changing to ODO in preference to standard tablets were the perception that ODO facilitates 
controlled intake and is easier to administer to uncooperative patients.

In Hori et al's study of first-episode schizophrenia patients $(\mathrm{N}=53)$ treated with ODO, a significant improvement in PANSS-Excited Component (PANSS-EC; comprising excitement, hostility, hallucinatory behavior, uncooperativeness, and poor impulse control) score was seen by day 3 and a significant improvement in PANSS-Complementary Items (comprising anger, difficulty in delaying gratification, and mood instability) score was seen by day $7 .{ }^{36}$ During the 4-week study, 71.6\% (38/53) of patients had responded (defined as $\geq 30 \%$ reduction in PANSS total score from baseline), not including five patients who dropped out. The authors noted that the results are consistent with studies which demonstrated SOT to be efficacious in the acute treatment of excitement and psychomotor agitation associated with schizophrenia.

\section{Comparative studies}

Two RCTs ${ }^{27,28}$ and four prospective cohort studies ${ }^{29-32}$ compared the efficacy of ODO with SOT. Additionally, one relatively small $(\mathrm{N}=38)$ retrospective open-label study utilized chronological sequential assignment of patients to ODO or SOT and used PANSS to compare the efficacy of these groups. ${ }^{35}$ The results of these studies suggest that improvements, or maintenance of improvements, in schizophrenia symptoms seen with ODO are similar to those with SOT. ${ }^{27-32,35}$ Another study compared ODO with liquid risperidone. ${ }^{33}$ These studies are discussed in more detail below.

In a conference presentation (not yet published), an Eli Lilly-sponsored prospective observational study compared ODO and SOT in an Australian cohort $(\mathrm{N}=104)$ of inpatients with schizophrenia and related disorders over the first week of treatment. ${ }^{29}$ The study found that ODO was preferentially prescribed to noncompliant patients ( $83 \%$ of ODO patients versus $55 \%$ of SOT patients). However, in contrast to other observational studies described below, there was no evidence that ODO was prescribed more often to more severely ill patients in this study. While significant improvements were observed in both groups over 1 week in all effectiveness measures (PANSS-negative symptoms, PANSS-EC, and CGI-Severity [CGI-S] scale), no significant differences were observed between the groups. This suggests that in a routine inpatient setting, the effectiveness of SOT and ODO formulations of olanzapine are comparable, even though ODO was preferentially prescribed to the more difficult-to-treat nonadherent patient group.

Czekalla et al investigated the effectiveness of ODO compared with SOT in a 2 -week prospective observational study of 456 patients predominantly with schizophrenia, schizotypal, or delusional disorder $(80.3 \%) .{ }^{30}$ Clinical improvements (CGI-Improvement) in both formulation groups were similar with improvements in $91.8 \%$ of the ODO group compared with $92.3 \%$ in the SOT group. However, as pointed out by Czekalla et al, interpretation of these data should take into consideration the higher proportion of severely ill patients (ODO: 64.4\%; SOT: 49.8\%) and patients displaying externally-directed aggression (ODO: 37.7\%; SOT: $16.4 \%$ ), who may be more difficult to treat, in the ODO cohort. Additionally, around $50 \%$ of patients overall received relatively high doses of olanzapine ( $\geq 20 \mathrm{mg} /$ day) by the end of the observation period, particularly in the ODO group $(59.1 \%)$.

Pascual et al's naturalistic study compared ODO with conventional oral antipsychotic medication (mostly haloperidol) over 6 hours in the treatment of 80 acutely agitated patients with psychotic disorders in an emergency department setting. ${ }^{13}$ In such a setting, any advantage may keep both healthcare providers and patients safer from the consequences of aggression. While both groups had statistically significant improvements from baseline in PANSS-EC, a significant difference favoring ODO was observed at 1 hour (but not thereafter). Significant improvement overall from baseline was also seen in Agitation Calmness Evaluation Scale scores for both groups, but not between groups at any time point up to 6 hours. Although there was no significant difference in the proportion of patients in each group requiring physical restraint, more patients receiving ODO (70\%) did not require a second medication compared with the conventional antipsychotic treatment group $(50 \% ; P=0.04)$. However, as discussed in a later section, it is important to bear in mind the nonsignificance of secondary comparisons, which may be underpowered, should be interpreted with caution, and should not necessarily imply equivalence.

A posthoc analysis of a prospective open-label study by Arranz et al measured core symptom improvement in never-treated first-episode psychotic patients chronologically assigned to either ODO or SOT for 6 weeks (19 patients in each group).$^{35}$ Relative to baseline, a significant decrease in PANSS total score was observed in both groups (mean decrease of $33.4 \pm 20$ in the SOT group and 36.6 \pm 19 in the ODO group). However, treatment type was not found to have a significant effect on change in PANSS score.

To date, only two head-to-head studies comparing ODO with another antipsychotic have been conducted. Hatta et al evaluated the efficacy and tolerability of ODO compared with risperidone oral solution in Japanese acutely agitated 
psychotic patients over a short duration (1 hour). ${ }^{33}$ The design of this study was somewhat unusual in its use of a pseudorandomization protocol where patients were allocated (chronologically) to ODO in the first month of recruitment and risperidone oral solution in the second month of recruitment, or to the equivalent antipsychotic if it had worked well for them previously. Patients were subsequently followed up in a relatively naturalistic way. No significant difference between the treatments groups was seen in improvement in clinical symptomatology as assessed by change in PANSS-EC or CGI-S. Nor was there any significant difference between the groups in the proportion of patients requiring a "rescue" intramuscular treatment due to worsening. No significant differences in patient satisfaction with treatment were observed. Thus, similar efficacy between the two treatments in the acute setting in this patient population was concluded.

In a similar study of agitated psychotic patients at an acute care psychiatric ward in Taiwan, Hsu et al followed 42 patients for 24 hours after random allocation to either ODO, intramuscular olanzapine, risperidone oral solution, or intramuscular haloperidol. They found that these treatments were similarly effective in the treatment of agitation over 24 hours based on a number of measures (PANSS-EC, Agitation Calmness Evaluation Scale, and CGI-S). However, they found that ODO and intramuscular olanzapine (but not risperidone oral solution) were more effective than intramuscular haloperidol in the early phase of treatment (up to 90 minutes) based on PANSS-EC. Moreover, while not statistically significant, ODO produced slightly more improvement, based on PANSS-EC, in this small sample than intramuscular olanzapine within the first hour. ${ }^{34}$

Kuramochi et al assessed a cohort of 1068 schizophrenia patients from a 6-week Japanese noninterventional study of olanzapine in which patients were stratified by formulation initially prescribed (ODO or SOT) ${ }^{31}$ They found that while ODO treatment was associated with a significantly shorter duration of illness (16.3 versus 18.4 years) and more severe acute phase symptoms for the current episode, positive symptoms of schizophrenia improved significantly over 6 weeks and to a similar degree regardless of which formulation was initially prescribed.

In the only randomized double-blind clinical trial comparing ODO and SOT, Karagianis et al administered ODO sublingually in a double-dummy fashion (that is, active standard tablet plus placebo ODT or placebo standard tablet plus active ODT) in order to preserve blinding to the active formulation. ${ }^{27}$ Although this study was predominantly designed to investigate the effects of continuing SOT treatment versus switching to ODO on body mass index (BMI) and weight in patients who had previously gained weight with SOT, a number of efficacy measures were also included in this 16-week study. No statistically significant differences between the treatment groups were observed in any efficacy measures (CGI-S, Global Assessment of Functioning scale, and Subjective Wellbeing Under Neuroleptics scale). However, it must be borne in mind that patients in both groups were clinically stable at baseline and so opportunity for improvement was limited.

The other randomized study was a 12 -week preference study of ODO and SOT in patients with schizophrenia conducted by Bitter et al. ${ }^{28}$ This study also included CGI-S as a measure of efficacy and found no significant difference in efficacy between the two formulations $(P=0.87)$ while adjusting for baseline adherence level. However, it should be noted that in order to be entered into the study, patients had to be stable and tended to have mild to moderate symptoms (CGI-S: ODO 2.3; SOT 2.5) which remained stable throughout the duration of the study.

The longest comparative study of ODO versus SOT (12 months) was conducted by Chartier et al, which evaluated the effectiveness of oral olanzapine formulations in a European cohort (Greece 38\%, France 35\%, and Germany $26 \%$ ) of patients with schizophrenia or bipolar disorder treated in a naturalistic setting. ${ }^{32}$ Patients initially prescribed ODO were significantly younger, had a significantly shorter history of illness, and were significantly more likely to be diagnosed with bipolar disorder. They were also significantly more severely ill, irrespective of disease type, and were rated significantly less adherent with medication at baseline than SOT-treated patients. These factors may explain why patients initiated on ODO were prescribed significantly higher doses of olanzapine on average (15.0 mg/day) compared with patients initiated with SOT (10.6 mg/day). As may be expected, ODO tended to be prescribed to patients with less insight into their illness, its effects, and need for treatment, suggesting that utilization of the two oral olanzapine formulations is associated with different patient profiles. However, regardless of indication, patients on ODO formulation experienced significantly greater improvements in disease severity, global functioning, and wellbeing (though not medication adherence and therapeutic alliance) compared with the SOT group.

\section{Safety}

As both ODO and SOT contain olanzapine as the active ingredient, they were expected to have similar safety and 
tolerability profiles. Indeed, the tolerability profile seen in clinical studies with ODO has been found to be similar to the more extensively studied SOT formulation. A number of studies of ODO have included measurement of safety and tolerability.

\section{Treatment-emergent adverse events (TEAEs)}

There are five studies reporting safety information on TEAEs with ODO. ${ }^{22,27,28,30,38}$ In a small pilot study by Chue et al, ${ }^{22}$ three of eleven patients had nonserious TEAEs including asthenia and insomnia. In a larger study by Kinon et $a 1,{ }^{38}$ TEAEs reported by at least $10 \%$ of patients included agitation, dry mouth, headache, insomnia, somnolence, and weight gain. However, while quantitatively and qualitatively similar to SOT studies, these single-arm studies did not allow direct comparisons of the adverse event profile of ODO with SOT.

In the 16-week double-blind RCT conducted by Karagianis et al, ${ }^{27}$ a similar proportion of patients in the SOT treatment arm developed TEAEs compared with patients in the ODO $\mathrm{arm}$. The most commonly reported adverse events (occurring in $\geq 5 \%$ of patients in either treatment arm) were increased appetite, headache, somnolence, fatigue, and dizziness. Two patients in the ODO arm developed serious adverse events, including one with dizziness requiring hospitalization and one who attempted suicide. As a double-blind trial, this is the most appropriate study design to investigate the comparative effect of treatment on safety and tolerability.

Adverse events were also assessed in a prospective comparative cohort study. ${ }^{30}$ In this study, $6.5 \%$ of patients on ODO developed adverse events compared with $2.9 \%$ of patients on SOT. Half of the adverse events in both arms were considered mild $(50.0 \%)$, while the majority of the remaining adverse events were considered moderate (46.4\%). There were three discontinuations due to adverse events in the ODO arm, caused by allergic reaction, increased creatine kinase, and restlessness. It is important to keep in mind that in this study, both clinicians and patients were aware of which treatment patients were taking. This has the potential to bias the reported rate of adverse events when new treatments are being used.

In the open-label crossover study by Bitter et al, ${ }^{28}$ which randomized patients to ODO or SOT, no significant difference was found in the proportion of patients experiencing at least one TEAE while treated with either medication (ODO: $16.8 \%$, SOT: $12.5 \% ; P=0.31$ ). The most common adverse events in the ODO group were weight increase (7.6\%), hypertriglyceridemia (2.4\%), and somnolence $(1.6 \%)$.
Both randomized studies have thus shown no difference between ODO and SOT in terms of frequency of TEAEs, ${ }^{27,28}$ while naturalistic studies, ${ }^{30}$ where treatment decisions are made by physicians on the basis of patient characteristics and in the course of normal clinical practice in the knowledge of the treatment being prescribed, have tended to show a higher rate of TEAEs with ODO. As suggested by Czekalla et al, ${ }^{30}$ this may simply reflect baseline differences between the treatment groups such as a higher rate of utilization of ODO in more severely ill and/or nonadherent patients due to its perceived benefits in this population. Such patients may, in turn, be prescribed commensurately higher doses of olanzapine or concomitant medications, be more prone to adverse events, and be more closely monitored or more likely to report adverse drug reactions. On the other hand, randomization, particularly when combined with blinding to treatment, is designed to negate such imbalances between patient characteristics in relation to treatment allocation, allowing for more objective comparison of treatment effects. Therefore, the differences in the rate of adverse events observed with different formulations in open-label naturalistic studies may reflect preexisting differences in characteristics of the patient groups being compared rather than differences in the safety profiles of ODO and SOT.

\section{Extrapyramidal symptoms}

Treatment-emergent movement disorders, including extrapyramidal symptoms and tardive dyskinesia, are associated with a number of antipsychotic medications and may have a significant impact on treatment nonadherence and discontinuation. ${ }^{42,43}$ In the single-arm open-label study by Kinon et al, significant improvement in extrapyramidal symptoms was observed in the ODO-treated patients after switching from SOT as measured by the Simpson-Angus Scale. ${ }^{38}$ A nonsignificant reduction as measured by the Barnes Akathisia Scale was also reported. In addition, patients showed a reduction in tardive dyskinesia as measured by the Abnormal Involuntary Movement Scale. However, it should be noted that patients in this study were able to switch to SOT after 1 week of ODO treatment if the treating physician felt that the patient had experienced significant improvement in psychosis and medication adherence, which occurred relatively frequently (as noted above, 24 patients switched to SOT while 49 patients remained on ODO). For this reason, the improvements noted above may not be directly attributable to the ODO formulation.

In contrast, Hori et al found no significant change in extrapyramidal symptoms over the course of their 4-week 
study, although there was a numerical decrease relative to baseline as measured by the Drug-Induced Extrapyramidal Symptoms Scale (DIEPSS). ${ }^{36}$ Similar to Hori et al, Pascual et al also found no significant differences between ODO and conventional therapy groups on extrapyramidal symptoms as measured by the Udvalg for Kliniske Undersøgelser Side Effect Rating Scale. No movement disorders were spontaneously reported by patients. ${ }^{13}$ Thus, overall, extrapyramidal symptoms have tended to decline in some ODO studies and not differed significantly when directly compared with SOT.

\section{Suicidality}

In Czekalla et al's study of ODO and SOT, ${ }^{30} 52.6 \%$ of patients had some degree of suicidal ideation at baseline as measured by the mean score of item ten on the Montgomery Asberg Depression Rating Scale. This included 6.6\% of patients with distinct suicidal plans. The proportion of patients with some degree of suicidal ideation was found to similarly decrease from baseline after 2 weeks of therapy for both treatment groups (SOT from $51.2 \%$ to $22.7 \%$; ODO from $53.9 \%$ to $20.6 \%$ ). No patients with distinct suicidal plans were observed after 2 weeks in either treatment group. In Karagianis et al's study, one patient (from the ODO group) attempted suicide. ${ }^{27}$ One completed suicide was reported by Bitter et al, although it was not reported which treatment group this patient belonged to at the time. ${ }^{28}$

\section{Weight gain and metabolic profile}

Weight gain and changes in metabolic parameters have been reported during treatment with atypical antipsychotics, including olanzapine. Patients should be monitored at baseline and periodically during treatment. Long-term studies of olanzapine ( $\geq 48$ weeks; 573 days median) have demonstrated a mean weight gain relative to baseline of $5.6 \mathrm{~kg} .{ }^{44}$ As mentioned earlier, ODO may be absorbed via the buccal mucosa or before reaching the pylorus (particularly when administered sublingually), and it has been hypothesized that this may lead to a different weight change profile compared with SOT. ${ }^{45}$ Further explanation of the hypothesis can be found in Karagianis et al's review. ${ }^{45}$

Some support, albeit limited, for the above hypothesis initially came from some small/pilot studies and case reports which have predominantly included younger males with schizophrenia, indicating greater weight gain or absence of weight loss with SOT relative to ODO. ${ }^{35,46-49}$ For example, Arranz et al observed an increase in mean body weight over 6 weeks of $6.3 \pm 1.9 \mathrm{~kg}$ in SOT-treated patients compared with only $3.3 \pm 3.2 \mathrm{~kg}$ in ODO-treated patients $(P=0.009) .{ }^{35}$
Substantial weight gain ( $\geq 7 \%$ increase from baseline weight) was observed in $84.2 \%$ of SOT patients and in $31.6 \%$ of ODO patients in this study. Potential differences in the risk of weight gain and changes in metabolic parameters during treatment with ODO compared to SOT may impact treatment choice and patient adherence.

Studying this potential difference in weight gain, a European research group recently conducted a small randomized crossover study in 12 healthy males to compare the shortterm metabolic profiles of ODO and SOT over 8 days. ${ }^{50}$ This study showed that although patients treated with olanzapine experienced changes in glucose and lipids, as well as weight gain, there were no significant differences between the ODO and SOT formulations in metabolic profile (homeostasis model of assessment of insulin resistance, fasting, and postprandial triglyceride and free fatty acid concentrations), body composition, fuel oxidation, or physical activity level, which may have explained potential differences in the weight gain profiles of the formulations. ${ }^{50}$ From this same study, it was also determined that patients treated with ODO and SOT had no consistent differences in changes in a variety of gut hormones that may influence body weight. ${ }^{51}$ Moreover, studying a cohort of 23 patients with bipolar depression, Bobo et al used a randomized open-label design to compare ODO with SOT with regard to weight, BMI, fasting lipid, glucose, insulin, and leptin concentrations as well as two measures of eating attitude (Food Craving Inventory and Three-Factor Eating Questionnaire) over a longer timeframe of 8 weeks. ${ }^{52}$ With the exception of significantly lower triglyceride concentration in the ODO group at week 8, no other significant differences in metabolic profile or attitude towards food between the two formulations was found. However, it should be noted that in these studies, as with others, steps were not dictated in the protocol to ensure sublingual/pregut absorption of olanzapine with the ODO formulation (for example, by prohibiting food and beverage consumption after administration). Thus under such circumstances, in some cases, olanzapine from ODO would be expected to reach the gut and behave in the same way as SOT. Therefore, validity of the hypothesis of possible weight differences being mediated by gut receptors remains uncertain.

Also, in contrast to the small supportive studies mentioned above, recent larger and more rigorous studies, while inconclusive, have not confirmed the hypothesis that ODO has a more favorable weight gain profile than SOT. In single-arm studies, weight gain with ODO has been similar to that seen with SOT. For example, patients in Kinon et al's study gained $3.0 \mathrm{~kg}$ on average over 6 weeks (although, as 
noted above, some of these patients switched to SOT after 1 week, limiting the usefulness of the data in comparing the weight gain profiles of the formulations $)^{38}$ and Hori et al found that weight (by $3.1 \mathrm{~kg}$ ), BMI, plasma levels of triglycerides, and total cholesterol significantly increased, ${ }^{36}$ although blood sugar levels did not significantly change. In Czekalla et al's 2-week study, ${ }^{30}$ weight gain was reported as an adverse event by more ODO patients (2.8\%) than SOT patients $(1.0 \%)$. Also, in Bitter et al's open-label crossover study, ${ }^{28}$ a similar degree of weight gain was observed for the two formulations $(0.6 \mathrm{~kg}$ and $0.8 \mathrm{~kg}$ for SOT and ODO, respectively). In Chartier et al's prospective observational study, no significant difference in weight gain was found between ODO and SOT over 12 months (schizophrenia: ODO $2.6 \mathrm{~kg}$ versus SOT $2.3 \mathrm{~kg}, P=0.42$; bipolar disorder: ODO $3.0 \mathrm{~kg}$ versus $2.5 \mathrm{~kg}, P=0.54) .{ }^{32}$

A study that was designed to compare BMI and weight gain with ODO and SOT in healthy subjects over 3 weeks was recently stopped prematurely due to poor enrollment. ${ }^{53}$ As a consequence of the small number of patients who enrolled and took medication (ODO: $\mathrm{N}=7$; SOT: $\mathrm{N}=9$ ), there was no opportunity for meaningful analysis. Thus, while only indicative, there was no evidence for significant group differences between ODO- and SOT-treated patients (ODO group gained $3.3 \mathrm{~kg}$ on average versus $3.2 \mathrm{~kg}$ for SOT group).

In an attempt to resolve the question of whether ODO has a more favorable weight gain profile than SOT, a rigorous double-blind double-dummy study was conducted by Karagianis et al. ${ }^{27}$ The PLATYPUS study, as it was named, failed (based on its primary endpoint) to confirm an advantage for ODO over SOT on body weight and BMI as there was no significant difference between the two formulations in change from baseline in these measures after 16 weeks (primary analysis); ODO group gained $1.42 \mathrm{~kg}$ on average versus $2.08 \mathrm{~kg}$ for SOT group $(P=0.385)$. Moreover, the number of patients experiencing substantial weight gain (ODO: $14.6 \%$, SOT: $11.1 \%$ ) was not significantly different between groups $(P=0.624)$. However, a significant group difference in adherence was observed whereby ODO patients were more likely to take their medication as indicated, which may have impacted the incidence of weight gain in the ODO group due to greater and more prolonged antipsychotic exposure. Moreover, significant differences were detected in favor of ODO for prespecified categorical weight change distribution (the majority of ODO-treated patients gained $\pm 1.0 \mathrm{~kg}$, whereas the majority of SOT-treated patients gained $1.5-3.5 \mathrm{~kg}$ ), and mean overall reduction in appetite (subjective measure by Visual Analog Scale) was significantly greater in ODO-treated patients. As pointed out by Karagianis et al, the analysis was not adjusted for these multiple secondary comparisons.

In order to investigate the possible confounding effect that adherence may have had on the original results, Karagianis et al used data from the subset of adherent patients from the PLATYPUS study to investigate factors associated with weight gain during olanzapine treatment. ${ }^{54}$ This study found that the rate of weight gain slowed significantly more in patients switched from SOT to ODO compared to those continuing with SOT. Also, consistent with previous openlabel findings, weight gain was lower in males (but not females) and patients residing in the US (but not in Mexico or Canada) treated with ODO, compared with those treated with SOT. Given the posthoc and unpowered nature of these analyses, these exploratory results should be interpreted with caution.

The currently available evidence regarding a difference in the potential for weight gain with ODO versus SOT is inconsistent and warrants further study. However, what does seem apparent is that if a difference exists between formulations in this regard, it is not likely to be a substantial one and may manifest only in certain patient populations. It should also be borne in mind that equivalence of weight gain during olanzapine treatment should not be an overriding consideration if it comes at the cost of lower adherence and potential relapse.

\section{Patient preference and medication adherence}

Patient preference is an important factor in long-term treatment adherence and thus treatment outcomes. Patients are more likely to persist with therapies they have had some active involvement in choosing, possibly aided by an enhanced therapeutic alliance as a consequence of improved patient motivation and insight into their illness and its treatment. $28,55,56$

One single-arm study provided data on patient acceptance. Patients in Kinon et al's study rated their acceptance of ODO using the Patient Global Impression Scale from one (I like it very much) to seven (I dislike it very much). ${ }^{38}$ Patientrated feelings about the medication showed positive acceptance of ODO at all measured time points (range in Patient Global Impression Scale scores: 2.01-2.74). Bitter et al also undertook an investigation into this important aspect of oral olanzapine formulation by comparing patients' preference for ODO versus SOT in a randomized open-label crossover study. ${ }^{28}$ The primary finding of this study was 
that significantly more patients preferred ODO over SOT. Overall, 61\% of patients preferred ODO, 27\% preferred SOT, and $12 \%$ expressed no preference. Even taking into account a significant sequence effect with patients tending to prefer the last formulation they received, this result remained highly significant $(P<0.001)$. In other words, while patients preferred the last formulation that they had taken, this preference was not strong enough to overcome the formulation preference in favor of ODO. Common free-text responses by patients as reasons for preference for a specific formulation were ease of use, taste, expectation of better effectiveness, and weight change.

Medication nonadherence is an issue of significant clinical importance in patients with serious mental illnesses such as schizophrenia and bipolar disorder. This is due to the fact that in these populations, the rate of nonadherence is high and is associated with poor outcomes such as low rates of remission and high rates of relapse, including hospitalization. ${ }^{57-62}$ For example, patients who discontinue their medications have been found to have a monthly relapse rate of $11 \%$, compared to that of $3.5 \%$ per month for patients who continue on maintenance treatment with conventional antipsychotic drugs. ${ }^{63}$ Consequently, long-term outcomes such as quality of life and comorbid conditions may also be negatively affected by treatment nonadherence. ${ }^{64-67}$

In general, olanzapine studies utilizing SOT formulation have shown it to have an advantage in terms of treatment adherence and persistence over a number of typical and atypical antipsychotics. ${ }^{7,8,67-71}$ Despite the risk of weight gain during treatment with olanzapine, this adherence advantage may be largely conferred through its relatively favorable efficacy. ${ }^{1,2,72-75}$ It has been demonstrated that better adherence and persistence on antipsychotic medication is associated not only with better improvements in symptom severity ${ }^{76}$ and a higher level of functioning, ${ }^{77}$ but also with a significantly lower risk of psychiatric hospitalization. ${ }^{59,78-80}$

It appears that ODO may have an additional adherence advantage over SOT. The 16-week randomized double-blind double-dummy study by Karagianis et al found a significantly higher proportion (92.9\%) of patients on ODO being adherent (based on a pill count of at least $75 \%$ relative to that prescribed) compared with $78.5 \%$ of patients on SOT $(P=0.015) .{ }^{27}$ Since this was a blinded double-dummy study, this finding cannot be explained by physical differences in formulation. Karagianis et al suggested that the difference may be due to ODO possibly having some favorable effect, such as less appetite increase (or satiety failure), resulting in patients wanting to take medication more regularly.
The findings of Karagianis et al are augmented by data from various studies, some of which were single-arm studies showing significant improvement in patients' adherence and/or attitudes toward medication intake with ODO. In a single-arm 6-week study of nonadherent patients who were switched to $\mathrm{ODO},{ }^{38}$ treatment adherence, as measured by the Treatment Compliance Interview and the nursing staff's Nursing Assessment of Medication Acceptance (NAMA) compliance scale, had significantly improved from baseline to endpoint. Moreover, patients' attitudes toward medication intake, per change in Rating of Medication Influences Noncompliance Score, also improved with a statistically significant reduction in this score within the first 2 days of therapy with ODO. Importantly, findings from these indirect and subjective measures of adherence (which may be unreliable), were supported by measurement of patients' olanzapine plasma concentration levels. ${ }^{38}$

There is additional evidence that treatment adherence may be further enhanced with ODO relative to SOT. Czekalla et al investigated the NAMA compliance scale as well as ingestion of ODO and SOT. They found that overall acceptance was lower for ODO at baseline (implying a potentially more noncompliant patient population to begin with), but ODO acceptance improved to a greater degree, almost reaching the same level as in SOT-treated patients, after 2 weeks. ${ }^{30}$

In Chartier et al's prospective observational study, almost all patients on either ODO or SOT (95.3\% on ODO, 93.7\% on SOT) completed the 12-month study, showing a similarly high persistence level on the medication. ${ }^{32}$ Although the two groups did not significantly differ regarding change from baseline in the Medication Adherence Rating Scale, ODO-treated patients were significantly less adherent to their antipsychotic medications at baseline and had less insight into their illness as measured by the Scale to Assess Unawareness of Mental Disorder.

In their preference study of ODO versus SOT, Bitter et al also assessed medication adherence with the Medication Adherence Form and found this measure to be similarly high for both formulations (ODO: 94\%; SOT: $93 \%$; defined as adherent based on a score of $>75 \%$ on the Medication Adherence Form).$^{28}$ This finding was supported by tablet count ( $>98 \%$ patients deemed compliant for both formulations). This study also found no significant differences between the treatment groups in change in attitude towards treatment, as measured by the Drug Attitude Inventory. Considering that patients included in this study had to be clinically stable and remained so throughout the study, this lack of significant difference in adherence may 
result from this being an inherently adherent population of patients to begin with, thus having only minimal opportunity for further improvement in this regard.

When study design and differences in patient populations being compared are taken into consideration, overall, the above results suggest ODO is associated with similar, and likely improved, adherence compared with SOT. As ODO was designed for, and is often used in, patients with suspected or verified adherence problems, this should be reassuring for clinicians and should be considered when treating patients where opening a "therapeutic window," in which greater insight and treatment adherence and/or persistence may lead to improved long-term outcomes, is a pivotal goal.

\section{Nursing burden}

Schizophrenia and bipolar disorder impose a significant work burden on nursing staff in inpatient treatment settings where treatment often needs to be administered under difficult circumstances and patients closely monitored on an ongoing basis. It would be expected that a treatment that is effective, well-tolerated, easy to administer, aids adherence, is well-accepted by patients, and whose administration can be verified would reduce nurses' workload. In order to systematically investigate this important aspect of psychiatric treatment and quantify various aspects of nursing resource utilization, Eli Lilly and Company developed the NAMA scale. This scale is completed by nursing staff and measures patients' acceptance and attitude towards medication, including burden of care on nursing staff. ${ }^{38}$ The NAMA questionnaire includes four items: (1) attitude (patient has a positive attitude towards medication), (2) compliance (patient complies with medication intake), (3) ingestion (patient ingests medication), and (4) nursing (no extensive nursing effort is needed to administer medication); a total score may also be calculated. Results relating to item two (compliance) were previously noted in the section on adherence. Due to its relevance to the intention of the ODO formulation, the scale has been used in a number of ODO studies.

Using NAMA, two single-arm studies have shown ODO to decrease nurses' workload. Kinon et al used the NAMA questionnaire to assess nursing burden of treatment with ODO in acutely ill noncompliant patients with schizophrenia and schizoaffective disorder. ${ }^{38}$ Significant improvements in nursing burden were seen as early as day 2 and improvements generally continued through to week 6 . Hori et al also used the NAMA questionnaire to assess nursing burden in first-episode schizophrenia. ${ }^{36}$ NAMA total score decreased from 11.3 at baseline to 7.9 at day $28(P<0.01)$, with a significant decrease seen as early as day 7. Consistent with these results, without using NAMA, Van Heeringen et al investigated whether the use of ODO had an impact on nursing workload or the need for intramuscular medication in a retrospective observational study in psychiatric inpatients and outpatients. They found that, based on physicians' judgment, use of ODO tablets reduced nurses' workload in 64.6\% of cases and intramuscular administration was avoided in $65.1 \%$ of cases. ${ }^{39}$

In their large prospective observational study, Czekalla et al used NAMA to assess nursing burden associated with ODO and SOT. ${ }^{30}$ Although a smaller proportion of patients showed positive medication acceptance towards ODO compared with SOT at the beginning of the study, a proportionally higher number of patients showed a positive acceptance towards ODO at 2 weeks, resulting in a similar level of positive medication acceptance in ODO and SOT groups by this time. Thus, there is some evidence to suggest that ODO may not only reduce nursing burden relative to no treatment or other antipsychotics, but also when compared with SOT.

\section{Clinical utility}

What conclusions of clinical relevance can then be drawn from the existing data? The studies reviewed above included a broad range of patient populations representative of those seen in usual practice settings, including those with first-episode schizophrenia, ${ }^{36}$ patients already stabilized on treatment, ${ }^{22,28}$ patients with diagnoses of schizophrenia, schizoaffective, schizophreniform disorder, and bipolar disorder, ${ }^{27,38}$ and patients who are known to be nonadherent with treatment. ${ }^{38}$ These data have shown ODO to be a useful addition to the standard oral tablet form of olanzapine which has similar pharmacokinetic, efficacy/effectiveness, tolerability, and characteristics. However, ODO formulation may have some advantages, not only where medical conditions present swallowing difficulties for the patient with standard tablets (eg, dysphagia, stroke, gastroesophageal reflux disease) and in situations where access to liquids or discretion is needed, but also where rapid effect (eg, agitated patients in emergency settings) and/or where medication adherence is considered to be a potential concern or medication administration needs to be verified. Data from open-label naturalistic studies, where physicians were free to prescribe according to their usual practice, also indicates that when given the choice of ODO or SOT, they often (with some exceptions) selected ODO for the treatment of more severely symptomatic patients. In regards to the important 
consideration of risk of weight gain during treatment with olanzapine, ODO may not have a significant advantage over SOT in all patients but at least does not appear to increase the risk of weight gain over SOT. Also, as there is a general preference amongst patients for ODO and it has been shown to reduce nurses' workload, other than a potential cost difference in some countries, there seems to be little disincentive to offering patients this formulation over SOT as a matter of standard practice. However, the choice of ODO over SOT would seem particularly prudent when there is a change in treatment setting which may impact adherence, such as moving from a more controlled environment to a less controlled one, eg, discharge from an inpatient facility or change in caregiver or accommodation.

\section{Limitations of existing ODO clinical data}

Although there are a number of atypical antipsychotics in orally disintegrating formulations (eg, clozapine, risperidone, aripiprazole), very little information is available for those medications. On the other hand, limited as it is relative to SOT, more substantive data from across patient populations, world geographies, study types, and study measures is available from ODO studies. However, a number of limitations, as well as strengths, of the existing data need to be acknowledged and taken into consideration.

With the exception of two large RCTs, the PLATYPUS study ${ }^{27}$ and Bitter et al's preference study, ${ }^{28}$ the existing data reviewed in this article arises mostly from open-label and/or nonrandomized naturalistic studies. Each study design has its advantages and disadvantages which need to be considered in the interpretation of these results. In the case of randomized trials, this often involves the inclusion of only certain types of patients who meet strict selection criteria and who are willing to consent to a rigid study protocol. Moreover, study conditions may not reflect real life practice conditions and, consequently, it may not be appropriate to generalize the results of such studies to the broader population with the disease state under investigation. This may be the case with the preference study of Bitter et al, ${ }^{28}$ where patients needed to be stabilized on medication prior to entering the study. Also, patients in the PLATYPUS study must have already been stable, taking SOT, and experienced significant weight gain. ${ }^{27}$

While naturalistic observational studies have few inclusion criteria and are conducted under usual practice conditions, they are more representative of the types of outcomes that are to be expected in typical clinical practice. However, as patients tend to be allocated to medication the investigator believes is best suited to them, this inevitably leads to imbalance in various characteristics associated with treatment decisions (some of which may be unmeasured or unobserved), and may impact the final outcomes. Thus, in some ODO studies, patients with more severe symptoms who were considered less likely to be adherent with medication tended to be prescribed ODO. In such cases, comparing potential treatment effects in disparate patient groups may be misleading, even though some attempt may be made to adjust for this baseline imbalance using statistical methods. This difficulty is further complicated by the generally unrestricted use of concomitant medications and switching of treatments in naturalistic studies which makes linking outcomes with particular treatments more tenuous.

Regarding the apparent overrepresentation of observational research relative to $\mathrm{RCT}$ data in this review, it is worth bearing in mind that although RCTs are the gold standard in establishing the efficacy and safety of new therapeutic agents, observational research plays a valid role in filling the gap in knowledge between efficacy in highly selected patient groups in rigorously monitored/stringent settings and effectiveness in usual practice settings. The latter provides important insights into how the treatment actually performs and interacts with various patient characteristics, comorbidities, and concomitant medications which are not necessarily present in clinical trials, yet is the environment in which the treatment will ultimately be used. This distinction is particularly important when considering the actual use and patient population which ODO could benefit (ie, those that would typically not be represented in RCTs). There would also be little value replicating the efficacy of ODO in highly controlled clinical trials when efficacy has already been established with SOT, as the active ingredient, olanzapine, is the same.

The main purpose of the ODO formulation has not been to alter the active ingredient but rather to enhance its palatability, ease of ingestion, convenience, and acceptability in patients, particularly those difficult-to-treat patients with swallowing problems or adherence issues. These aspects of pharmacological treatments are elusive to conventional RCT designs as they either exclude patients with such issues (despite their prevalence in the community) or are overridden by the study design (patients who do not take the medication as directed are usually discontinued/excluded from analysis and so factors such as convenience and patient preference are largely ignored). It is thus entirely understandable and to be expected that, with the exception of the PLATYPUS study which aimed to answer a very specific question, the focus of ODO research has not been the use of randomized 
clinical trials and replicating efficacy findings, but rather in the conduct of observational research to gain a better understanding of the benefits and limitations of the formulation in the actual target population under realistic conditions.

Another feature of the reviewed studies that complicates interpretation of the results is the tendency to conduct a multitude of secondary analyses, most of which were not sufficiently powered to assess the question at hand. Thus, nonsignificant results of the secondary analyses may arise from type II (false negative) error, whereas differences deemed significant on this basis may be consequence of type I (false positive) error.

Overall, while these studies provide a wealth of valuable information, in many cases the findings need to be replicated independently before they can be accepted as conclusive.

\section{Conclusion}

The literature reviewed in this article regarding the physical characteristics, efficacy/effectiveness, tolerability, and safety profile of ODO lend support to this formulation being a useful alternative to SOT, particularly in situations where patient adherence is problematic or verification of ingestion is needed, where the patient's ability to swallow standard tablets is compromised, and where other formulations (eg, injection) are resisted or considered undesirable. The existing data suggests that although having a similar efficacy and tolerability profile to SOT, long-term patient outcomes may be improved with ODO in some patients due to better adherence, leading to lower relapse/hospitalization risk. ODO may also have benefits over SOT in terms of nursing burden and patient preferences, and this should be taken into consideration when making treatment decisions. While other rapidly dissolving generic formulations of olanzapine are available in various countries around the world, given the ostensibly important role of physical characteristics in determining patient preference and adherence, the recent comparative dissolution study of Hobbs et $\mathrm{al}^{26}$ suggests that the majority of findings (other than bioequivalence) reviewed here may not necessarily be extrapolated to these other formulations.

In summary, taking into consideration the known efficacy and tolerability profile of olanzapine combined with the greater adherence potential of ODO, apparently influenced by patient preference considerations and rapid onset of efficacy which further enhances insight and patient acceptance of medication, this formulation is not only suitable for the majority of patients, but may be particularly useful in opening a "medication gateway" to some difficult-to-treat nonadherent patients.

\section{Disclosure}

WM, TT, JK, HAS, and GH are employees of Eli Lilly and Company, manufacturer of olanzapine in standard tablet form $\left(\right.$ Zyprexa $\left.^{\circledR}\right)$ and orally disintegrating form (Zyprexa ${ }^{\circledR}$ Zydis $^{\circledR} /$ Velotab $\left.^{\text {TM }}\right)$.

\section{References}

1. Leucht S, Corves C, Arbter D, Engel RR, Li C, Davis JM. Secondgeneration versus first-generation antipsychotic drugs for schizophrenia: a meta-analysis. Lancet. 2009;373(9657):31-41.

2. Leucht S, Komossa K, Rummel-Kluge C, et al. A meta-analysis of head-to-head comparisons of second-generation antipsychotics in the treatment of schizophrenia. Am J Psychiatry. 2009;166(2):152-163.

3. Tohen M, Treuer T. Clinical usefulness of olanzapine in bipolar disordera developer's insight. US Psyc. 2009;2(1):32-36.

4. Strom BL, Eng SM, Faich G, et al. Comparative mortality associated with ziprasidone and olanzapine in real-world use among 18,154 patients with schizophrenia: the Ziprasidone Observational Study of Cardiac Outcomes (ZODIAC). Am J Psychiatry. 2011;168(2):193-201.

5. McEvoy JP, Lieberman JA, Perkins DO, et al. Efficacy and tolerability of olanzapine, quetiapine, and risperidone in the treatment of early psychosis: a randomized, double-blind 52-week comparison. Am J Psychiatry. 2007;164(7):1050-1060.

6. Kahn RS, Fleischhacker WW, Boter H, et al. Effectiveness of antipsychotic drugs in first-episode schizophrenia and schizophreniform disorder: an open randomised clinical trial. Lancet. 2008;371(9618): 1085-1097.

7. Lieberman JA, Stroup TS, McEvoy JP, et al. Effectiveness of antipsychotic drugs in patients with chronic schizophrenia. $N$ Engl J Med. 2005;353(12):1209-1223.

8. Haro JM, Suarez D, Novick D, Brown J, Usall J, Naber D. Three-year antipsychotic effectiveness in the outpatient care of schizophrenia: observational versus randomized studies results. Eur Neuropsychopharmacol. 2007;17(4):235-244.

9. Karagianis J, Rosenbluth M, Tohen M, et al. Reviewing CATIE for clinicians: balancing benefit and risk using evidence-based medicine tools. Curr Med Res Opin. 2007;23(10):2551-2557.

10. Corrigan PW, Liberman RP, Engel JD. From noncompliance to collaboration in the treatment of schizophrenia. Hosp Community Psychiatry. 1990;41(11):1203-1211.

11. Lacro JP, Dunn LB, Dolder CR, Leckband SG, Jeste DV. Prevalence of and risk factors for medication nonadherence in patients with schizophrenia: a comprehensive review of recent literature. $J$ Clin Psychiatry. 2002;63(10):892-909.

12. Cooper D, Moisan J, Grégoire JP. Adherence to atypical antipsychotic treatment among newly treated patients: a population-based study in schizophrenia. J Clin Psychiatry. 2007;68(6):818-825.

13. Pascual JC, Pérez V, Martín JL, Safont G, Puigdemont D, Alvarez E. Olanzapine orally-disintegrating tablet in severe psychotic agitation: a naturalistic study. Actas Esp Psiquiatr. 2007;35(1):47-51.

14. American Psychiatric Association. Practice Guideline for the Treatment of Patients with Schizophrenia. 2nd ed. Arlington, VA: American Psychiatric Association; 2004.

15. Canadian Psychiatric Association. Clinical practice guidelines. Treatment of schizophrenia. Can J Psychiatry. 2005;50(13 Suppl 1):7S-57S.

16. Baloush-Kleinman V, Levine SZ, Roe D, Shnitt D, Weizman A, Poyurovsky M. Adherence to antipsychotic drug treatment in earlyepisode schizophrenia: a six-month naturalistic follow-up study. Schizophr Res. 2011;130(1-3):176-181.

17. Johnson D, Rai S, Milne S. Olanzapine oro-dispersible (Velotab) - an alternative to depot? Int J Psychiatry Clin Pract. 2002;6(3):163-165.

18. San L, Casillas M, Ciudad A, Gilaberte I. Olanzapine orally disintegrating tablet: a review of efficacy and compliance. CNS Neurosci Ther. 2008;14(3):203-214. 
19. Bogner RH, Wilkosz MF. Fast dissolving tablets: new dosage convenience for patients. US Pharm. 2002;27:34-43.

20. Kuchekar BS, Badhan AC, Mahajan HS. Mouth dissolving tablets: a novel drug delivery system. Pharma Times. 2003;35:7-9.

21. Seager $\mathrm{H}$. Drug-delivery products and the Zydis fast-dissolving dosage form. J Pharm Pharmacol. 1998;50(4):375-382.

22. Chue P, Jones B, Taylor CC, Dickson R. Dissolution profile, tolerability, and acceptability of the orally disintegrating olanzapine tablet in patients with schizophrenia. Can J Psychiatry. 2002;47(8):771-774.

23. Bergstrom RF, Mitchell M, Witcher J, et al. Rapid onset of absorption with olanzapine orally disintegrating tablets. J Emerg Nurs. 2004;30(5):416-417.

24. Witcher JW, Bergstrom RF, Cerimele BJ, et al. Pharmacokinetics and bioequivalence of olanzapine rapidly-disintegrating tablets. Pharm Sci. 1998;1(1):S487.

25. Markowitz JS, DeVane CL, Malcolm RJ, et al. Pharmacokinetics of olanzapine after single-dose oral administration of standard tablet versus normal and sublingual administration of an orally disintegrating tablet in normal volunteers. J Clin Pharmacol. 2006;46(2):164-171.

26. Hobbs D, Karagianis J, Treuer T, Raskin J. An in vitro analysis of disintegration times of different formulations of orally disintegrating olanzapine. Poster presented at: 10th World Congress of Biological Psychiatry; May 29-June 2, 2011. Prague, Czech Republic.

27. Karagianis J, Grossman L, Landry J, et al. A randomized controlled trial of the effect of sublingual orally disintegrating olanzapine versus oral olanzapine on body mass index: the PLATYPUS study. Schizophr Res. 2009;113(1):41-48.

28. Bitter I, Treuer T, Dilbaz N, et al. Patients' preference for olanzapine orodispersible tablet compared with conventional oral tablet in a multinational, randomized, crossover study. World J Biol Psychiatry. 2010; 11(7):894-903.

29. Damodaran S, D'Souza R, Katz P, et al. An Australian observational study of olanzapine tablet and wafer formulations for the treatment of inpatients with schizophrenia. Paper presented at: 8th Biennial Australasian Schizophrenia Conference; September 22-24, 2004. Brisbane, Australia.

30. Czekalla J, Wagner T, Schacht A, Kluge M, Kinon B. Effectiveness and medication acceptance of olanzapine disintegrating tablets compared to standard olanzapine tablets in acutely treated psychiatric patients. Patient Prefer Adherence. 2007;1:19-27.

31. Kuramochi M, Nishiuma S, Tanji U, Fujikoshi S, Fuchigami Y, Takahashi M. Actual state of use of orally disintegrating olanzapine tablets in patients with acute-stage schizophrenia. Rinsho Seishin Yakuri. 2009;12:2159-2168.

32. Chartier F, Rouillon F, Lukasiewicz M, Kraemer S, D'yachkova Y. Effectiveness of olanzapine coated and orally disintegrating tablets in a 1-year European observational study. Eur Neuropsychopharmacol. 2010;20(Suppl 3):S508-S509.

33. Hatta K, Kawabata T, Yoshida K, et al. Olanzapine orally disintegrating tablet vs risperidone oral solution in the treatment of acutely agitated psychotic patients. Gen Hosp Psychiatry. 2008;30(4):367-371.

34. Hsu WY, Huang SS, Lee BS, Chiu NY. Comparison of intramuscular olanzapine, orally disintegrating olanzapine tablets, oral risperidone solution, and intramuscular haloperidol in the management of acute agitation in an acute care psychiatric ward in Taiwan. $J$ Clin Psychopharmacol. 2010;30(3):230-234.

35. Arranz B, San L, Dueñas RM, et al. Lower weight gain with the orally disintegrating olanzapine than with standard tablets in first-episode never treated psychotic patients. Hum Psychopharmacol. 2007;22(1): $11-15$.

36. Hori H, Ueda N, Yoshimura R, et al. Olanzapine orally disintegrating tablets (Zyprexa Zydis) rapidly improve excitement components in the acute phase of first-episode schizophrenic patients: an open-label prospective study. World J Biol Psychiatry. 2009;10(4 Pt 3):741-745.

37. Dardennes R, Chartier F, Heurtebize N, Olivier V, Perrin E. Naturalistic use of the orally disintegrating tablet formulation of olanzapine in acute schizophrenic patients: an observational prospective study. Int $J$ Neuropsychopharmacol. 2004;7(Suppl 2):P01-P389.
38. Kinon BJ, Hill AL, Liu H, Kollack-Walker S. Olanzapine orally disintegrating tablets in the treatment of acutely ill non-compliant patients with schizophrenia. Int J Neuropsychopharmacol. 2003;6:97-102.

39. Van Heeringen C, Vandendriessche F, Van Vleymen B, Gillain B. The effect of using orally disintegrating olanzapine tablets on agitation, cooperation and nurses' workload in psychiatric patients. Acta Psychiatr Belg. 2006;106:14-19.

40. Kay SR, Fiszbein A, Opler LA. The positive and negative syndrome scale (PANSS) for schizophrenia. Schizophr Bull. 1987;13(2):261-276.

41. Guy W. National Institute of Mental Health. ECDEU Assessment Manual for Psychopharmacology. Rockville, MD: US Department of Health, Education, and Welfare; 1976.

42. Pierre JM. Extrapyramidal symptoms with atypical antipsychotics: incidence, prevention and management. Drug Saf. 2005;28(3):191-208.

43. Perkins DO. Predictors of noncompliance in patients with schizophrenia. J Clin Psychiatry. 2002;63(12):1121-1128.

44. Citrome L, Holt RI, Walker DJ, Hoffmann VP. Weight gain and changes in metabolic variables following olanzapine treatment in schizophrenia and bipolar disorder. Clin Drug Investig. 2011;31(7):455-482.

45. Karagianis J, Hoffmann VP, Arranz B, et al. Orally disintegrating olanzapine and potential differences in treatment-emergent weight gain. Hum Psychopharmacol. 2008;23(4):275-281.

46. de Haan L, van Amelsvoort T, Rosien K, Linszen D. Weight loss after switching from conventional olanzapine tablets to orally disintegrating olanzapine tablets. Psychopharmacology (Berl). 2004;175(3): 389-390.

47. Stip E, Anselmo K, Wolfe M, Lessard C, Landry P. Long-term treatment with atypical antipsychotics and risk of weight gain. Drug Saf. 2006;29(6):550-552.

48. Crocq MA, Guillon MS, Bailey PE, Provost D. Orally disintegrating olanzapine induces less weight gain in adolescents than standard oral tablets. Eur Psychiatry. 2007;22(7):453-454.

49. Chawla B, Luxton-Andrew H. Long-term weight loss observed with olanzapine orally disintegrating tablets in overweight patients with chronic schizophrenia. A 1 year open-label, prospective trial. Hum Psychopharmacol. 2008;23(3):211-216.

50. Vidarsdottir S, Vlug P, Roelfsema F, Frölich M, Pijl H. Orally disintegrating and oral standard olanzapine tablets similarly elevate the homeostasis model assessment of insulin resistance index and plasma triglyceride levels in 12 healthy men: a randomized crossover study. J Clin Psychiatry. 2010;71(9):1205-1211.

51. Vidarsdottir S, Roelfsema F, Streefland T, Holst JJ, Rehfeld JF, Pijl H. Short-term treatment with olanzapine does not modulate gut hormone secretion: olanzapine disintegrating versus standard tablets. Eur $J$ Endocrinol. 2010;162(1):75-83.

52. Bobo WV, Epstein RA Jr, Shelton RC. Effects of orally disintegrating vs regular olanzapine tablets on body weight, eating behavior, glycemic and lipid indices, and gastrointestinal hormones: a randomized, open comparison in outpatients with bipolar depression. Ann Clin Psychiatry. 2011;23(3):193-201.

53. Hoffmann VP, Case M, Jacobson JG, Francis JL. Listing of metabolic changes in healthy volunteers receiving orally dissolving olanzapine or oral olanzapine: data from a clinical study that was terminated early. Schizophr Res. 2009;115(2-3):370-371.

54. Karagianis J, Landry J, Hoffmann VP, et al. An exploratory analysis of factors associated with weight change in a 16-week trial of oral vs orally disintegrating olanzapine: the PLATYPUS study. Int J Clin Pract. 2010;64(11):1520-1529.

55. Kassirer JP. Incorporating patients' preferences into medical decisions. New Engl J Med. 1994;330(26):1895-1896.

56. Jahng KH, Martin LR, Golin CE, DiMatteo MR. Preferences for medical collaboration: patient-physician congruence and patient outcomes. Patient Educ Couns. 2005;57(3):308-314.

57. Sullivan G, Wells KB, Morgenstern H, Leake B. Identifying modifiable risk factors for rehospitalization: a case-control study of seriously mentally ill persons in Mississippi. Am J Psychiatry. 1995;152(12): 1749-1756. 
58. Valenstein M, Copeland LA, Blow FC, et al. Pharmacy data identify poorly adherent patients with schizophrenia at increased risk for admission. Med Care. 2002;40(8):630-639.

59. Law MR, Soumerai SB, Ross-Degnan D, Adams AS. A longitudinal study of medication nonadherence and hospitalization risk in schizophrenia. J Clin Psychiatry. 2008;69(1):47-53.

60. Ascher-Svanum H, Zhu B, Faries DE, Furiak NM, Montgomery W. Medication adherence levels and differential use of mental-health services in the treatment of schizophrenia. BMC Res Notes. 2009;2:6.

61. Ascher-Svanum H, Zhu B, Faries DE, et al. The cost of relapse and the predictors of relapse in the treatment of schizophrenia. BMC Psychiatry. 2010;10:2

62. Novick D, Haro JM, Suarez D, Perez V, Dittmann RW, Haddad PM. Predictors and clinical consequences of non-adherence with antipsychotic medication in the outpatient treatment of schizophrenia. Psychiatry Res. 2010;176(2-3):109-113.

63. Weiden PJ, Olfson M. Cost of relapse in schizophrenia. Schizophr Bull. 1995;21(3):419-429.

64. Haywood TW, Kravitz HM, Grossman LS, Cavanaugh JL Jr, Davis JM, Lewis DA. Predicting the "revolving door" phenomenon among patients with schizophrenic, schizoaffective, and affective disorders. Am J Psychiatry. 1995;152(6):856-861.

65. Hunt GE, Bergen J, Bashir M. Medication compliance and comorbid substance abuse in schizophrenia: impact on community survival 4 years after a relapse. Schizophr Res. 2002;54(3):253-264.

66. Swanson JW, Swartz MS, Elbogen EB. Effectiveness of atypical antipsychotic medications in reducing violent behavior among persons with schizophrenia in community-based treatment. Schizophr Bull. 2004;30(1):3-20.

67. Ascher-Svanum H, Zhu B, Faries D, Landbloom R, Swartz M, Swanson J. Time to discontinuation of atypical versus typical antipsychotics in the naturalistic treatment of schizophrenia. BMC Psychiatry. 2006;6:8

68. Ascher-Svanum H, Zhu B, Faries DE, Lacro JP, Dolder CR, Peng X. Adherence and persistence to typical and atypical antipsychotics in the treatment of schizophrenia. Patient Prefer Adherence. 2008;2:67-77.

69. Beasley CM Jr, Stauffer VL, Liu-Seifert H, Taylor CC, Dunayevich E, Davis JM. All-cause treatment discontinuation in schizophrenia during treatment with olanzapine relative to other antipsychotics: an integrated analysis. J Clin Psychopharmacol. 2007;27(3):252-258.
70. Novick D, Ascher-Svanum H, Zhu B, et al. The number needed to treat for all-cause medication discontinuation in the treatment of schizophrenia: consistency across world geographies and study designs. Pharmacopsychiatry. 2010;43(3):81-85.

71. Haro JM, Novick D, Suarez D, Roca M. Antipsychotic treatment discontinuation in previously untreated patients with schizophrenia: 36-month results from the SOHO study. J Psychiatr Res. 2009;43(3):265-273.

72. Beasley CM Jr, Tollefson G, Tran P, Satterlee W, Sanger T, Hamilton S. Olanzapine versus placebo and haloperidol: acute phase results of the North American double-blind olanzapine trial. Neuropsychopharmacology. 1996;14(2):111-123.

73. Breier A, Berg PH, Thakore JH, et al. Olanzapine versus ziprasidone: results of a 28 -week double-blind study in patients with schizophrenia. Am J Psychiatry. 2005;162(10):1879-1887.

74. Dossenbach M, Pecenak J, Szulc A, et al. Long-term antipsychotic monotherapy for schizophrenia: disease burden and comparative outcomes for patients treated with olanzapine, quetiapine, risperidone, or haloperidol monotherapy in a pan-continental observational study. J Clin Psychiatry. 2008;69(12):1901-1915.

75. Kane JM, Osuntokun O, Kryzhanovskaya LA, et al. A 28-week, randomized, double-blind study of olanzapine versus aripiprazole in the treatment of schizophrenia. J Clin Psychiatry. 2009;70(4):572-581.

76. Liu-Seifert H, Adams DH, Kinon BJ. Discontinuation of treatment of schizophrenic patients is driven by poor symptom response: a pooled posthoc analysis of four atypical antipsychotic drugs. BMC Med. 2005;3:21.

77. Dunayevich E, Ascher-Svanum H, Zhao F, et al. Longer time to antipsychotic treatment discontinuation for any cause is associated with better functional outcomes for patients with schizophrenia, schizophreniform disorder, or schizoaffective disorder. J Clin Psychiatry. 2007;68(8): 1163-1171.

78. Weiden PJ, Kozma C, Grogg A, Locklear J. Partial compliance and risk of rehospitalization among California Medicaid patients with schizophrenia. Psychiatr Serv. 2004;55(8):886-891.

79. Svarstad BL, Shireman TI, Sweeney JK. Using drug claims data to assess the relationship of medication adherence with hospitalization and costs. Psychiatr Serv. 2001;52(6):805-811.

80. Thieda P, Beard S, Richter A, Kane J. An economic review of compliance with medication therapy in the treatment of schizophrenia. Psychiatr Serv. 2003;54(4):508-516.
Patient Preference and Adherence

\section{Publish your work in this journal}

Patient Preference and Adherence is an international, peer-reviewed, open access journal focusing on the growing importance of patient preference and adherence throughout the therapeutic continuum. Patient satisfaction, acceptability, quality of life, compliance, persistence and their role in developing new therapeutic modalities and compounds to

\section{Dovepress}

optimize clinical outcomes for existing disease states are major areas of interest. This journal has been accepted for indexing on PubMed Central. The manuscript management system is completely online and includes a very quick and fair peer-review system. Visit http://www.dovepress.com/ testimonials.php to read real quotes from published authors. 